\title{
"Building Hope for the Future": A Program to Foster Strengths in Middle-School Students
}

\author{
Susana C. Marques $\cdot$ Shane J. Lopez $\cdot$ J. L. Pais-Ribeiro
}

Published online: 10 December 2009

(C) Springer Science+Business Media B.V. 2009

\begin{abstract}
The authors investigated the effectiveness of a 5-week hope-based intervention designed to enhance hope, life satisfaction, self-worth, mental health and academic achievement in middle school students. The study includes a sample of 31 students from a community school, a matched comparison group of 31 students, and 2 secondary groupsguardians and teachers of the students' intervention group. Students completed a questionnaire packet that included demographic information, the Portuguese versions of the Children's Hope Scale, Students' Life Satisfaction Scale, Mental Health and Self-Worth Scales. Academic achievement was obtained from school records. At baseline, groups are statistically similar on the variables of interest. At post-test the intervention group had enhanced hope, life satisfaction and self-worth. In the intervention group, benefits in hope, life satisfaction and self-worth were maintained at the 18-month follow up. Results suggest that a brief hope intervention can increase psychological strengths, and participants continue to benefit up to 1-year and 6-months later.
\end{abstract}

Keywords Academic achievement · Intervention - Mental-health · Middle-schoolers · Positive thinking variables

\section{Introduction}

In Snyder's hope theory, hope has gone beyond wishful thinking to an understanding of how intentional thought leads to adaptive action. Snyder et al. (1991) characterized hope as a human strength manifested in capacities to: (a) clearly conceptualize goals (goals thinking), (b) develop the specific strategies to reach those goals (pathways thinking), and (c) initiate and sustain the motivation for using those strategies (agency thinking).

S. C. Marques $(\bowtie) \cdot$ J. L. Pais-Ribeiro

Department of Psychology and Educational Sciences, University of Porto,

Rua do Dr. Manuel Pereira da Silva, 4200-392 Porto, Portugal

e-mail: dscmarques@mail.telepac.pt

The Clifton Strengths School and Gallup, 1001 Gallup Drive, Omaha, NE 68102, USA 